\title{
Threat Assessment Of Medicinal Plants Of Koria District In Chhattisgarh (India).
}

\author{
Mantosh Kumar Sinha \\ K.R. Technical College , Ambikapur (Sarguja University Ambikapur) Chhattisgarh, (India).
}

\begin{abstract}
Koria district of Chhattisgarh state has a very rich plant diversity specially of medicinal plants and there is no comprehensive description of the flora as well as vascular cryptogames of the district is available some plant species are on the verge of extinction..The objective of the study was threat assessment of medicinal plants of Koria district based on the criteria developed by the IUCN.

Keeping these point in view the present investigation was planned to assess the biobiversity of the vegetation of the district.Extensive field surveys were undertaken during 2004-08 Sampling sites were selected randomly covering all the blocks. Quantitative phytosociological characters were studied as per methods described by Misra (1968). The threat categories in the present study have been prepared viewing to the medicinal plants found in the wild only. Assessment of threat was done following the guidelines of IUCN (2000)

Study reveals that 3 medicinal plant taxa were found to be regionally extinct in wild(Ex.), 14 were critically endangered(CR), 27 were endangered (EN),62 were vulnerable (VU) and 37 were near threatened $(N T)$ and 57 were least concerned $(L C)$.
\end{abstract}

Key Words:-, Threat assessment,Koria district, Medicinal plants

\section{Introduction}

India contains about $8 \%$ of world's biodiversity on $2 \%$ of the earth's surface, making it one of the 12 mega diversity countries in the world.

Chhattisgarh, the $26^{\text {th }}$ state of the country, has ample variation in physical and cultural features. It has about $44 \%$ of its total geographical area covered with forests

Koria district in Chhattisgarh lies between $22^{0} 58^{\prime}$ and $23^{0} 51^{\prime}$ North Latitude and $81^{0} 59^{\prime}$ and $82^{0} 45^{\prime}$ East Longitude and has a forest area of $81.23 \%$. Average rainfall is $121.36 \mathrm{~cm}$. and annual mean temperature is $24^{0} \mathrm{c}$. The district is dominated by Upper Gondwana rocks which are rich in deposition of coal.

The district has a sizeable tribal population using enormous range of plants for their basic needs, sustenance and livelihood. The district has very rich plant diversity, including medicinal plants.

Many of them are on the verge of extinction due to over exploitation and destruction of their habitat. There has been no comprehensive study on the enumeration, distribution and the assessment of threat to the existing medicinal plants.

Keeping these points in view the present investigation was planned to assess the diversity of the medicinal plants. Attempts have also been made to assess the threat status and the extent of damage to medicinal plants of the Koria district.

\section{Material and methods}

Extensive field survey were undertaken during the years 2004 to 2008.

Covering an area of $20 \mathrm{~km}$ to $200 \mathrm{~km}$. radius around the head quarter town of the koria district. The district comprises of 5 Development Blocks, viz. Baikunthpur, Sonhat, Manendragarh, Khadgawan and Bharatpur. The sampling sites were selected randomly covering all the blocks.These include Shivpur, Katghodi, Pahadpara, Tilpandand, Itga, Rakiya, deori ,Orgai, Salgawan, Amhar Narayanpur, Khongapani, Parasgadi, Udalkachhar Podidih, Peeparbahra, Jilda, Bhagwanpur,Patwahi and semaria . Medicinal plants has been collected ,herbarium sheets were prepared identified using standard flora and references. The quantitative phytosociological characters were studied using methods described by Misra (1968).

To assass the threat status guidelines of FRLHT (Foundation for Revitalisation of Local Health Traditions), Bangalore (2003) have been followed and the data thus computed have been expressed in terms of IUCN threat categories. 


\section{Observation and Results}

Two hundred eighty four genera distributed in 93 families were showing the $31 \%$ of the total Medicinal plants of state. Evaluation of status of threat reveals that 3 medicinal plant taxa were found to be regionally extinct in wild(Ex), 14 were critically endangered(CR), 27 were endangered (EN),62 were vulnerable (VU) and 37 were near threatened (NT), 57 were least concerned(LC).

\section{Discussion} the country.

There are several reports of the floristic diversity and region threat assessment from many regions of

As per the 3rd Conservation Assessment \& Management Plan Workshop for Southern Indian Medicinal Plants held at Bangalore in January, 1997, some endemic, rare, critically endangered and vulnerable floral species in the State of Chhattisgarh were listed. The endemic and rare species of Chhattisgarh state include Sophera bakeri, Crotalaria trifoliustrum (Khip), Uraria prunellaefolia (Pitharan), Mucuna imbricata (Kevanch), Hoya wrighitii, Desmodium tortosum (Sarivan) and Erythrina resupimata (Pangra).

Among 45 endangered taxa (based on floristic study of 7 districts of Chhattisgarh by NBRI, Lucknow) some of the plant species are Butea monosperma var. lutea, Celastrus paniculata, Chlorophytum tuberosum, $C$. arudinaceum, Clerodendron serratum, Cordia rothii, Curculigo orchioides, Curcuma aromatica, Pterocarpus marsupium, Rauwolfia serpentina. In the present study,it was found that, Curculigo orchioides, Rauwolfia serpentina, Cordia macleodii were critically endangered Costus speciosus and Chlorophytum tuberosum, Clerodendron serratum were endangered plant species

Varghese ,etal. (1999) reported the ecological niches and amplitudes of rare, threatened and endemic trees of Peppara Wildlife Sanctuary. They have documenting 151 tree species belonging to 51 families with 62 endemics ( $41 \%$ of endemism), 6 rare and 8 threatened species.

Mandal et al. (2000) reported rare and endangered flowering plants of Bay Islands with special reference to endemics and extra-Indian taxa. They concluded that 110 plant species are considered as rare threatened

Samant, et.al (2003) studied diversity and conservation status of medicinal plants in Uttaranchal state. They have noted that Critically Endangered 18 spp., Endangered 18 spp., Vulnerable 22 spp., Low-risk Near Threatened 6 spp. and Low-risk Least Concern 1 sp.

Badola, et.al (2003) have emphasized threatened medicinal plants and their conservation in Himachal Himalayas. They analysed 133 rare, sensitive and threatened medicinal plant species of Himachal Himalayas.

Jaipuriar (2003)has also dealt with the threatened herbal flora of Jharkhand. he has reported 8 species have been listed as endangered 18 species as vulnerable .Strychnos nux vomica (Kuchala) of family Loganiaceae, Caryota urens (Mari) of Palmae and Sarcostemma acidum (Somlata) have been assessed as extinct(Ex.)

Mathachen (2004) studied ecological amplitude and regeneration of medicinally important threatened trees in the central Western Ghats. They surveyed out of total, 19 species of medicinally important threatened trees were recorded.

Sukumaran et al. (2005) carried out the floristic composition of Sacred Groves as a functional tool to analyse the mini forest ecosystem. The study has overall record of flora of the 329 species enumerated 54 are listed rare, endemic and threatened.

Arvind et al. (2005) reported a comparative analysis of red-listed and non-red-listed plant species in the Western Ghats, India. They have recorded at both the regional and the local scales.

Udayan (2006) reported a few rare, endemic and red-listed species from high range (Mannavanshola), near Munnar in Idukki district (Kerala state). He collected 25 rare, endemic and red-listed species.

Laloo et al. (2006) studied status of medicinal plants in the disturbed and undisturbed sacred forests of Meghalaya. . They have explored Camellia caduca (endemic and less frequent), Cinnamomum pauciflorum (endemic and rare), Erythroxylum kunthianum (endemic) and Picrasma javanica (rare) were studied. 
Maliya (2007) presented a local assessment of rare species of Katarniya ghat wildlife sanctuary, Dist. Bahraich, Uttar Pradesh, India. Fortyone rare species have been recorded in the sanctuary region by him.

Patil (2007) studied conservation of medicinal plants through people's participation - A case study of Toranmal, Maharashtra. He concluded that out of 24 medicinal plants found in this area, 16 were endangered, vulnerable or threatened.

Sukumaran and Raj (2007) reported rare, endemic, threatened (RET) trees and lianas in the Sacred groves of Kanyakumari district. The study enlisted 36 RET species from 21 families, belonging to 29 genera. Out of thirtysix, 23 are endemic to Western Ghats and Tamilnadu at present

Dubey et al. (2007) have assessed a total of 313 plant species of Rewa (M.P.). They have categorized 3 species as regionally "Extinct in the wild", 25 as "Critically endangered", 59 as "Endangered", 97 as "Vulnerable" and 46 as "Not threatened" but at lower risk.

Reddy et al. (2008) have published first Red List of medicinal plants of Andhra Pradesh (India) in "Ethnobotanical leaflets". Out of total 50 prioritised medicinal plant species found in Andhra Pradesh, 39 were found to be in threatened group. The Red list status was assigned as 'Critically Endangered' 4, 'Endangered' 24, and 'Vulnerable' 11.

A comparison with IUCN status (Wikipedia 2009) the taxa assessed in the present study show that there is an increase of 14 species in Critically endangered category, 71 species in Endangered category and a decrease of 01 species in Vulnerable category, 25 in Near threatened category and 56 in Least concerned category.

\section{Conclusion}

It is concluded that 03 species as regionally "Extinct in the wild", 25 as "Critically endangered", 59 as "Endangered", 97 as "Vulnerable" and 46 as "Not threatened" but at lower risk

\section{Acknowledgement}

I am thankful to Dr. (Mrs.) Veenapani Dubey Head. Department of Botany C.M.D.P.G.College , Bilaspur (C.G.) for her keen interest and expert guidance

I am also thankful to local medicinemen and villagers of Koria district who generously contributed their knowledge and assisted me in my data collection .

\section{References}

[1] Varghese, A.O. and Menon, A.R.R. ,Ecological niches and amplitudes of rare threatened and endemic trees of Peppara Wildlife Sanctuary. Curr. Sci. Vol. 76 (9) ,1999, 1204-1208.

[2] Mandal, A.B.; Chattopadhyay and Coomar Tarun., Rare and endangered flowering plants of Bay islands with special reference to endemics and extra Indian taxa. Indian Forester, Vol. 126 (4) ,2000,389-396.

[3] Samant, S.S. and Mohinder Pal ,Diversity and conservation status of medicinal plants in Uttaranchal state. Indian Forester 129(9)2003,1090-1108.

[4] Badola, K. Hemant and Mohinder Pal, Threatened Medicinal plants and their conservation in Himachal Himalayas. Indian Forester, 129(1) 2003,55-68.

[5] Jaipuriar, M.K. ,Threatened herbal heritage of tribal land $\quad$ Jharkhand. Indian Forester, $\quad$ Vol. 129 (1) $2003,48-54$.

[6] Mathachen, Georgi P.; R. Vasudeva, H.C. Hombe Gowda, K.N. Ganeshaiah and R. Uma Shankar, Ecological amplitude and regneration of medicinally important threatened trees in the central Western Ghats. Indian Forester 130(11),2004,1330-1338.

[7] Sukumaran, S.; G.S. Rejini Balasingh, A. Kavitha and A.D.S. Raj ,The floristic composition of Sacred Groves: A functional tool to analyse the mini forest ecosystem. Indian Foreser 131(6) ,2005,773-785.

[8] Arvind, N.A., J. Manjunath, Dinesh Rao, K.N. Ganeshaiah, Uma R. Shanker, and G. Vanaraj Are red-listed species threatened? A comparative analysis of red-listed and non-red-listed plant species in the Western Ghats, India, Curr. Sci., 88(2), 2005, 258265.

[9] Udayan, P.S. ,Notes on a few rare, endemic and red-listed species from High Range (Mannavanshola) near Munnar, Idukki district, Kerala state. Indian Forester 132(11),2006, 1453-1464.

[10] Laloo, R.C.; L. Kharlukhi, S. Jeeva and B.P. Mishra ,Status of medicinal plants in the disturbed and the undisturbed sacred forests of Meghalaya, north-east India: Population structure and regeneration efficacy of some important species. Curr. Sci. 90(2) 2006, 225-232.

[11] Maliya, S.D. Rare species of Katarniyaghat Wildlife Sanctuary, District Bahraich, Uttar Pradesh,India Indian Forester, Vol. 133(8) , 2007,1052-1056.

[12] Patil, S.H. ,Conservation of medicinal plants through people's participation - A case study of Toranmal, Maharashtra. Indian Forester 133(3),2007,301-305.

[13] Sukumaran, S. and A.D.S. Raj, Rare, endemic, threatened (RET) trees and lianas in the Sacred groves of Kanyakumari district. Indian Forester 133(9),2007,1254-1266.

[14] Dubey, P.C., S.N. Mishra, Arjun Tiwari ,Loss of biodiversity with reference to important medicinal plants in Vindhya region and their threat assessment. Jou. of Trop. Forestry, 23(I\&II) 2007, 105-127. 
Threat Assessment Of Medicinal Plants Of Koria District In Chhattisgarh (India).

[15] Reddy, K.N. and C. Sudhakar Reddy ,First Red List of Medicinal plants of Andhra Pradesh, India. Conservation assessment and management planning. Ethnobotanical Leaflets 12,2008, 103-107.

[16] Mishra, R.,Ecology Workbook. (Oxford and IBH Publication Co., New Delhi ,1968) 244.

\section{TABLE:-1 Regional Threat Status of Medicinal Plants of Koria} district C.G.(India)

\section{Regionally Extinct in Wild (Ex.):-}

\begin{tabular}{|l|l|l|l|}
\hline$\underline{\text { S.No. }}$ & \multicolumn{1}{|c|}{ Botanical Name } & Vernacular name & Family \\
\hline$\underline{1}$ & $\begin{array}{l}\text { Curcuma amada } \text { Roxb } \\
\text { Kapurahalad }\end{array}$ & $\begin{array}{l}\text { Ambahaldi,Banhaldi, Amada, } \\
\text { Zingiberaceae }\end{array}$ \\
\hline$\underline{2}$ & Elaeocarpus ganitrus Roxb & Rudraksha & Tiliaceae \\
\hline$\underline{3}$ & Manihot glaziovii L. & Rubbe & Euphorbiaceae \\
\hline
\end{tabular}

\section{Critically Endangered (CR.) :-}

\begin{tabular}{|c|c|c|c|}
\hline$\underline{\text { S.No }}$ & Botanical Name & Vernacular name & Family \\
\hline$\overline{11}$ & Bixa orellana & Sinduri & Bixaceae \\
\hline 2 & Celastrus paniculata Willd & $\begin{array}{c}\text { Malkangini, Malkoni, Maltangun, } \\
\text { Umigini, Jyotismati }\end{array}$ & Celastraceae \\
\hline 3 & $\begin{array}{l}\text { Cordia macleodii Hook.f. } \\
\& \text { Thomson }\end{array}$ & Dahiman, Dahipalas, Dhengan & Boraginaceae \\
\hline 4 & Croton tiglium $\mathrm{L}$ & $\begin{array}{c}\text { Jamalghota, Dantichhoti, Triphals, } \\
\text { Hakani, Dantibeej }\end{array}$ & Euphorbiaceae \\
\hline 5 & Embelia robusta Roxb. & $\begin{array}{c}\text { Baibrang, Waiwarang, Bebrang, } \\
\text { Bhingi, Baberang }\end{array}$ & Myrsinaceae \\
\hline 6 & Grewia tiliaefolia Vahl. & Dhaman & Tiliaceae \\
\hline 7 & Leea macrophylla Horn. & Hatkan & Ampelidaceae \\
\hline 8 & Lepidium sativum $\mathrm{L}$. & Chandrashoor, Halim, Alevari & Brassicaceae \\
\hline 9 & Litsea glutinosa Lour & Maida lakdi, Menda & Lauraceae \\
\hline 10 & Oroxylum indicum Vent & Sauna, Sonpatta, Sonapatha, Arlu & Bignoniaceae \\
\hline 11 & Rauwolfia serpentina Benth & Sarpgandha & Apocynaceae \\
\hline 12 & Tecomella undulata Seem & Rakta Rohna & Bignoniaceae \\
\hline 13 & $\begin{array}{ll}\text { Curculigo } & \text { orchioides } \\
\text { Gaertn } & \\
\end{array}$ & Kali musli & Amaryllidaceae \\
\hline 14 & Prosopis spicigera L. & Shami & Mimosaceae \\
\hline
\end{tabular}

\section{Endangered (EN) :-}

\begin{tabular}{|l|l|l|l|}
\hline S.No & Botanical Name & Vernacular name & Family \\
\hline 1 & Acacia concinna Dc & Sikakai, Chikakai, Kochi, Ritha & Mimosaceae \\
\hline 2 & Acorus calamus L & Buch, Ghorbuch, Barja, Gorbach & Araceae \\
\hline 3 & . Adiantum lunulatum Burm. & Hansraj, Hansiya, Dakul & Polypodiaceae \\
\hline 4 & Aristolochia indica L. & Ishwarmul & Aristolochiaceae \\
& & & \\
\hline 5 & Buchanania lanzan Spreng & Char, Chiranji & \\
\hline 6 & Chlorophytum tuberosum Baker & Safe musli & Anacardiaceae \\
\hline
\end{tabular}


Threat Assessment Of Medicinal Plants Of Koria District In Chhattisgarh (India).

\begin{tabular}{|c|c|c|c|}
\hline 7 & Clerodendron serratum Spreng & Bharangi & Verbenaceae \\
\hline 8 & Costus speciosus Smith & Keo-Kanda & Zingiberaceae \\
\hline 9 & Crinum asiaticum $\mathrm{L}$. & Amaryllidaceae & Amaryllidaceae \\
\hline 10 & $\begin{array}{l}\text { Cymbopogon flexuosus } \\
\text { Hack. }\end{array}$ & Lemon Grass & Poaceae \\
\hline 11 & Embelia ribes Burm. & Baibidang, Lamjak, Lamjay, Khavi & Myrsinaceae \\
\hline 12 & Gardenia lucida Roxb & $\begin{array}{l}\text { Deekamali, } \quad \text { Kamari, } \\
\text { Nadihing }\end{array}$ & Rubiaceae \\
\hline 13 & Gloriosa superba L. & $\begin{array}{cc}\text { Kaliyari, } & \text { Kolihari, } \\
\text { Shankarpushpi, } & \text { Kalihari }\end{array}$ & Liliaceae \\
\hline 14 & Gymnema sylvestre R.Br. & Gurmar, Madhunashini, Ajgandhini & e $\quad$ Asclepiadacea \\
\hline 15 & Hedychium coronarium Koenig & Gulbakawali & Zingiberaceae \\
\hline 16 & $\begin{array}{l}\text { Lasiosiphon eriocephalus } \\
\text { Decne }\end{array}$ & Ramaitha, Dajdharuha, Ramatta & Thymelaeaceae \\
\hline 17 & Pandanus odoratissimus Roxb & Ketki, Keura, Keora & Pandanaceae \\
\hline 18 & Passiflora incarnata $\mathrm{L}$. & $\begin{array}{l}\text { Kaurav Pandav, Prempushpi, } \\
\text { Shivposh, Ghadiphoo }\end{array}$ & Passifloraceae \\
\hline 19 & Piper longum L. & Pipli, Pipari, magham, Peeparmool & Piperaceae \\
\hline 20 & Plumbago zeylanica L. & Chiutar, Chitrak & Plumbaginaceae \\
\hline 21 & Pterocarpus marsupium Roxb. & Bija, Paisar, Bijasal & Papilionaceae \\
\hline 22 & Rauwolfia tetraphylla $\mathrm{L}$ & Bada Chandrica & Apocynaceae \\
\hline 23 & Terminalia chebula Retz & Harra, Harad & Combretacea \\
\hline 24 & Uraria picta Desv & Pristiparni, Prashnaparni, Pitvan & Papilionaceae \\
\hline 25 & Withania somnifera Dun. & Ashwagandha, Asgandh, Rasbhari & Solanaceae \\
\hline 26 & Artemisia maritima Linn & Davana, Kirmala & Asteraceae \\
\hline 27 & Cryptolepsis buchanani Roem & Nagbel, Karanta & Asclepiadaceae \\
\hline
\end{tabular}

Vulnerable (VU):-

\begin{tabular}{|c|c|c|c|}
\hline S.No & Botanical Name & Vernacular name & Family \\
\hline$\overline{1}$ & $\overline{\text { Abelmoschus moschatus } \mathrm{L} .}$ & $\overline{\text { Kasturi bhindi, Jangli bhindi }}$ & Malvaceae \\
\hline 2 & Abrus precatorius $\mathrm{L}$. & Patha, Ratti, Gunja, Gumchi & Papilionaceae \\
\hline 3 & Adina cordifolia Hook. f. & Haldu, Karam & Rubiaceae \\
\hline 4 & Alstonia scholaris Brown & $\begin{array}{l}\text { Saptparni, Chhitwan, Satona, } \\
\text { Satwan }\end{array}$ & Apocynaceae \\
\hline 5 & $\begin{array}{ll}\text { Amorphophallus campanulatus } \\
\text { Blume }\end{array}$ & Suran Kanda, Ol, Olna & Araceae \\
\hline 6 & Anamirta Cocculus W. \& A. & $\begin{array}{l}\text { Kakmari, Jarmeh, Netramal, } \\
\text { Huber }\end{array}$ & Menispermaceae \\
\hline 7 & Andrographis paniculata Nees & $\begin{array}{lll}\text { Patthar } & \text { Neem, } & \text { Chiretta, } \\
\text { Bhuinim } & & \\
\end{array}$ & Acanthaceae \\
\hline 8 & Anona reticulata $\mathrm{L}$ & Ramphal, Barhial & Anonaceae \\
\hline 9 & Anthocephalus cadamba Miq & Kadamba & Rubiaceae \\
\hline 10 & Asparagus racemosus Willd & Satawar, Satawari, Satmuli, & Liliaceae \\
\hline 11 & Barleria prionitis $\mathrm{L}$. & Katsariya, Kantaphool & Acanthaceae \\
\hline
\end{tabular}


Threat Assessment Of Medicinal Plants Of Koria District In Chhattisgarh (India).

\begin{tabular}{|c|c|c|c|}
\hline 12 & Bauhinia vahlii W. \& A. & Mahul & Caesalpiniaceae \\
\hline 13 & Bryonopsis laciniosa $\mathrm{L}$ & Shivlingi & Cucurbitaceae \\
\hline 14 & . Caesalpinia bonducella Flem. & Katkaranj, Karanjuwa, Gataine & Caesalpiniaceae \\
\hline 15 & Cannabis corniculata $\mathrm{L}$ & Kandamool & Cannabinaceae \\
\hline 16 & Centella asiatica $\mathrm{L}$ & Brahmi, Kotyali, Birhami & Apiaceae \\
\hline 17 & Cinnaтотит tamala Fr. Nees. & Tejpatta, Tejpal, Patraj & Lauraceae \\
\hline 18 & Cissus quadrangularis L. & Harzor, Harjora, Hadjod & Ampelidaceae \\
\hline 19 & Clitoria ternatea $\mathrm{L}$. & $\begin{array}{l}\text { Aparajita, } \\
\text { Vishnukanta }\end{array}$ & Papilionaceae \\
\hline 20 & $\begin{array}{lll} & \text { Convolvulus pluricaulis } \\
\text { Chois } & & \end{array}$ & Shankh pushpi & Convolvulaceae \\
\hline 21 & Cordia myxa $\mathrm{L}$ & Lasoda & Boraginaceae \\
\hline 22 & Curcuma angustifolia Roxb. & Tikhur, Tikari & e $\quad$ Zingiberacea \\
\hline 23 & Curcuma aromatica Salisb. & Jangli haldi, Vanhaldi & e Zingiberacea \\
\hline 24 & Cyperus scariosus $\mathrm{Br}$ & Nagarmotha & Cyperaceae \\
\hline 25 & Desmodium gangeticum D.C & $\begin{array}{l}\text { Salparni, Galphulli, Acjariya, } \\
\text { Serivan }\end{array}$ & Papilionaceae \\
\hline 26 & Dioscorea bulbifera L. & Varahikand & Dioscoreaceae \\
\hline 27 & Dioscorea daemona Roxb & Baichandi & Dioscoreaceae \\
\hline 28 & Eulophia campestris Wall. & Saalmishri, Salibmisri & Orchidaceae \\
\hline 29 & Ficus glomerata Roxb & Gular, Paroa, Daduri, Kakmal & Moraceae \\
\hline 30 & Ficus infectoria $\mathrm{Roxb}$ & Pakar, Prarohi & Moraceae \\
\hline 31 & Flemingia chappar $\mathrm{Ham}$ & Gulphulli, Golaphuli & Papilionaceae \\
\hline 32 & Flemingia nana Roxb & Balraj & Papilionaceae \\
\hline 33 & Fumaria parviflora Lamk. & Pitpapra, Bag Gajar, & Fumariaceae \\
\hline 34 & Glossogyne pinnatifida DC & Tejraj & Asteraceae \\
\hline 35 & Grewia hirsuta Vanb & Gudasakri, Nagbela & Tiliaceae \\
\hline 36 & Hemidesmus indicus $\mathrm{Br}$. & $\begin{array}{l}\text { Anantmool, Kalisar, Kalidudha, } \\
\text { Shyamlata, } \\
\text { Krishnasariw }\end{array}$ & Asclepiadaceae \\
\hline 37 & Hymenodictyon excelsum Wall & $\begin{array}{l}\text { Bhawarmaal, Potar, Barita } \\
\text { Kanda }\end{array}$ & Rubiaceae \\
\hline 38 & Hyptis suaveolens Poit & Bantulsa, Gangatulsi & Labiatae \\
\hline 39 & Ipomoea mauritiana Lam & $\begin{array}{l}\text { Patalkohda, } \quad \text { Bhukumda, } \\
\text { Badkakanda }\end{array}$ & Convolvulaceae \\
\hline 40 & Lannea grandis Roxb. & Gurja & Anacardiaceae \\
\hline 41 & Martynia diandra Glox. & Baghnakhi & Pedaliaceae \\
\hline 42 & Melia azedarach $\mathrm{L}$ & Bakain & Meliaceae \\
\hline 43 & Mucuna prurita Hook & $\begin{array}{l}\text { Kewach, Kamach, Konch, } \\
\text { Khajehra, } \\
\text { Kawachhu }\end{array}$ & Papilionaceae \\
\hline 44 & Murraya koenigii Spreng. & $\begin{array}{l}\text { Gandhela, Meetha Neem, } \\
\text { Barsanga }\end{array}$ & Rutaceae \\
\hline 45 & Nictanthes arbor-tristis L. & Harsingar, Parijat, Kharsalu & Oleaceae \\
\hline 46 & Operculina turpethum $\mathrm{L}$. & Nishoth & Convolvulaceae \\
\hline 47 & Oroxylum indicum Vent & Sauna, Sonpatta, Sonapatha & Bignoniaceae \\
\hline
\end{tabular}


Threat Assessment Of Medicinal Plants Of Koria District In Chhattisgarh (India).

\begin{tabular}{|l|l|l|l|}
\hline 48 & Pedalium murex L & Gokharu-bada, Gokara & Pedaliaceae \\
\hline 49 & Picrorhiza kurroa Benth & Kutki & Scrophulariaceae \\
\hline 50 & Pterospermum acerifolium Willd. & Kanakchampa, Kathchampa, & Sterculiaceae \\
\hline 51 & Pueraria tuberosa DC. & $\begin{array}{l}\text { Pathal Kohnra, Pathal } \\
\text { Bankumra }\end{array}$ & Papilionaceae \\
\hline 52 & Schleichera trijuga Willd & Kusum, Kasma & Sapindaceae \\
\hline 53 & Semecarpus anacardium L. & Bhilawa, Bhilama, Bhelwa & Anacardiaceae \\
\hline 54 & Sida spinosa L. & Nagbel, Bariar & Malvaceae \\
\hline 55 & Smilax zeylanica L. & Sherkand, Ramdatoon & Liliaceae \\
\hline 56 & Spilanthes acmella L & Akarkara, Akalkahra & Asteraceae \\
\hline 57 & Sterculia urens Roxb. & Kullu, Gulu, Kulli, & Sterculiaceae \\
\hline 58 & Stevia rebaudiana Bertoni & Mithi buti & Asteraceae \\
\hline 59 & Tectona grandis L. & Sagon, Sagwan & Verbenaceae \\
\hline 60 & Thalictrum foliolosum DC. & Mamiri, Piyaranga, Pinjari, & Ranunculaceae \\
\hline 61 & Tinospora cordifolia Miers & Giloe, Gurach & Menispermaceae \\
\hline 62 & Vitex negundo L. & Nirgundi, Nigori & Verbenaceae \\
\hline
\end{tabular}

\section{Near Threatened (NT) :-}

\begin{tabular}{|c|c|c|c|}
\hline S.No & Botanical Name & Vernacular name & Family \\
\hline$\overline{1}$ & $\overline{\text { Abutilon indicum } \mathrm{G} \text {. Don }}$ & Kanghi, Kakahi, Kaghai, & Malvaceae \\
\hline 2 & Acacia catechu Willd & Khair, Kheri, Khadir & Mimosaceae \\
\hline 3 & Amomum subulatum Roxb. & Bari Ilaichi & Scitaminaceae \\
\hline 4 & Bacopa monnieri Linn. & Nir Brahmi & Scrophulariaceae \\
\hline 5 & Barleria cristata Linn & Vajradanti & Acanthacea \\
\hline 6 & Boerhaavia diffusa $\mathrm{L}$ & Punarnava & Nyctaginaceae \\
\hline 7 & Cassia glauca Lamk & Jamrasi, Kalamuka, Bakra & Caesalpiniaceae \\
\hline 8 & Clerodendron infortunatum Gaertn & Ghentu, Bhant & Verbenaceae \\
\hline 9 & Cochlospermum religiosum DC & Kuraya, Gulgal, Kumbi & Cochlospermaceae \\
\hline 10 & Colebrookia oppositifolia Smith & Kala Bansa & Labiatae \\
\hline 11 & Dalbergia latifolia Roxb. & Kala Sheesham, Sitsal & Papilionaceae \\
\hline 12 & Dryopteris crenata Christ & Sarkhas,Kildaru,Vishora & Polypodiaceae \\
\hline 13 & Eclipta alba Hassk & Bhringraj, Keshraj, Bhangra & Asteraceae \\
\hline 14 & Erythrina indica Lamk & Pharhada, Dhawal, Dhak & Papilionaceae \\
\hline 15 & Euphorbia neriifolia $\mathrm{L}$ & Sehund & Euphorbiaceae \\
\hline 16 & Ficus hispida $\mathrm{L}$. & Kathumar, kathgular, Dumar & Moraceae \\
\hline 17 & Garcinia india $\mathrm{L}$. & Kokam, Pahada, Dancera, & Guttiferaceae \\
\hline 18 & Gmelina arborea Roxb & Khamhar, Gamari, Gambhar & Verbenaceae \\
\hline 19 & Helicteres isora $L$. & Marodphalli, Eaithi & Sterculiaceae \\
\hline 20 & Holarrhena antidysenterica Wall & Kutaj, Indrayan, Koreya & Apocynaceae \\
\hline 21 & Kaempferia rotunda $\mathrm{L}$. & Bhui Champa & Zingiberaceae \\
\hline 22 & Leucas cephalotes Spreng & Dhrodpushpi, Guma, Goma & Labiatae \\
\hline 23 & Mimosa pudica $\mathrm{L}$. & Lajwanti, Lamjak, Lamjay & Mimosaceae \\
\hline 24 & Mimusops hexandra Roxb. & Khirni & Sapotaceae \\
\hline 25 & Ougeinia dalbergioides Benth. & Tinsa, Tinis, Chhadan & Papilionaceae \\
\hline 26 & Oxalis corniculat $\mathrm{L}$. & $\begin{array}{l}\text { Teenpatiya, Changeri, } \\
\text { Khatkal }\end{array}$ & Geraniaceae \\
\hline 27 & Paederia foetida $\mathrm{L}$ & Gandhali & Rubiaceae \\
\hline
\end{tabular}


Threat Assessment Of Medicinal Plants Of Koria District In Chhattisgarh (India).

\begin{tabular}{|l|l|l|l|}
\hline 28 & Pongamia pinnata Pierre & Karanj, Kanja & Papilionaceae \\
\hline 29 & Premna integrifolia L & Arni, Aeran, ganibhari & Verbenaceae \\
\hline 30 & Psoralea corylifolia DC. & Bakuchi, Bavchi, & Papilionaceae \\
\hline 31 & Randia dumetorum Lamk. & Mehnar,madan, Menphal & Rubiaceae \\
\hline 32 & Salmalia malabaricum DC & Semal & Bombacaceae \\
\hline 33 & Solanum indicum L. & Kanteri Badi, Barhanta & Solanacea \\
\hline 34 & Symplocos racemosa Roxb. & Lodh & Styraceae \\
\hline 35 & Terminalia arjuna W. \& A & Arjuna & Combretaceae \\
\hline 36 & Terminalia belerica Roxb & Bahera & Combretaceae \\
\hline 37 & Xanthium strumarium Linn. & Gokhru, Gokhara, & Asteraceae \\
\hline
\end{tabular}

Fig. 1 :- THREAT ASSESSMENT OF MEDICINAL PLANTS OF KORIA DISTRICT IN CHHATTISGARH (INDIA)

Fig. -1 Category-wise Analysis of Threat

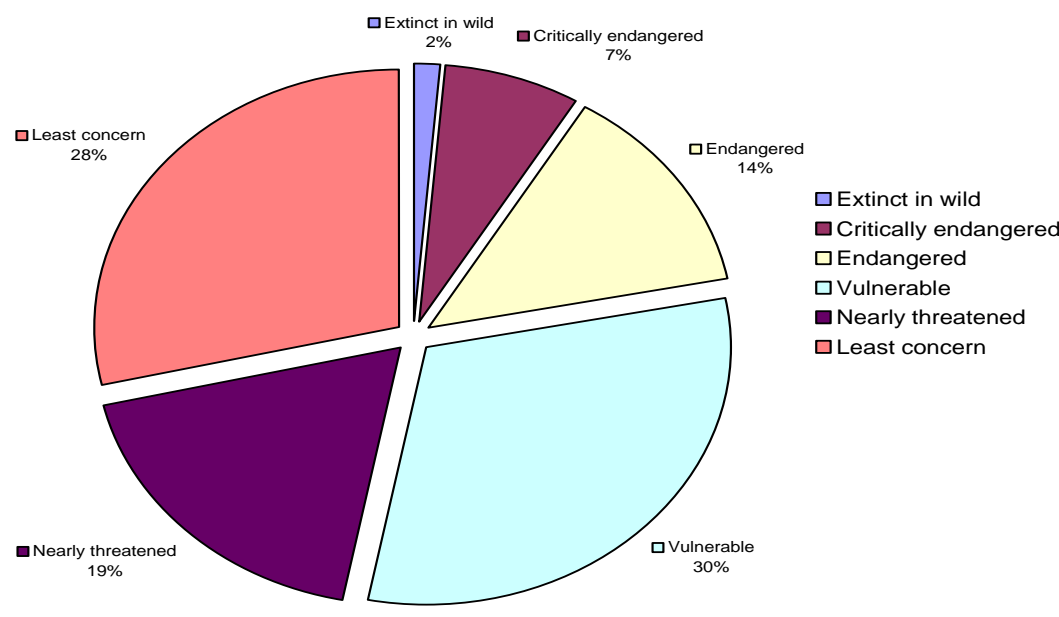

